$7^{\text {th }}$ Jagna International Workshop (2014)

International Journal of Modern Physics: Conference Series

Vol. 36 (2015) 1560012 (9 pages)

(C) The Authors

DOI: 10.1142/S2010194515600125

\title{
Critical slowing down in a dynamic duopoly
}

\author{
M. G. O. Escobido \\ W. Sycip Graduate School of Business, Asian Institute of Management, \\ 123 Paseo de Roxas, Makati City, 1229, Philippines \\ mescobido@aim.edu \\ www.aim.edu \\ N. Hatano \\ Institute of Industrial Science, University of Tokyo, \\ Komaba 4-6-1, Meguro, Tokyo 153-8505, Japan \\ hatano@iis.u-tokyo.ac.jp
}

Published 2 January 2015

\begin{abstract}
Anticipating critical transitions is very important in economic systems as it can mean survival or demise of firms under stressful competition. As such identifying indicators that can provide early warning to these transitions are very crucial. In other complex systems, critical slowing down has been shown to anticipate critical transitions. In this paper, we investigate the applicability of the concept in the heterogeneous quantity competition between two firms. We develop a dynamic model where the duopoly can adjust their production in a logistic process. We show that the resulting dynamics is formally equivalent to a competitive Lotka-Volterra system. We investigate the behavior of the dominant eigenvalues and identify conditions that critical slowing down can provide early warning to the critical transitions in the dynamic duopoly.
\end{abstract}

Keywords: Critical slowing down; Lotka-Volterra; complex systems.

\section{Introduction}

There has been active research to identify signals that can provide early warning prior to critical transitions across different systems. ${ }^{1,2}$ Prominent among them is the observation that as the system approaches a critical point, it becomes increasingly slow in recovering from small perturbations. Mathematically, this means that the characteristic return time will increase when one approaches the critical point. ${ }^{3}$ This phenomenon has been shown to occur across varied fields such as semiconductor lasers, ${ }^{4}$ engineered systems ${ }^{5}$ and financial markets. ${ }^{6}$ The intent of the paper is to

This is an Open Access article published by World Scientific Publishing Company. It is distributed under the terms of the Creative Commons Attribution 3.0 (CC-BY) License. Further distribution of this work is permitted, provided the original work is properly cited. 
add industrial firms among systems where critical slowing down may anticipate transitions.

Anticipating critical transitions is of fundamental interest in economic systems. For this complex system, changes are not necessarily smooth but can be abrupt, and may cause a critical transition from one dynamical state to another. For instance, the market crash in 1929 brought with it two thousand investment firms and resulted in a structural change in the American banking industry. ${ }^{7}$ The more recent financial crisis pushed the global economy into a great recession for which many nations are still reeling from. ${ }^{8}$

Analyzing critical transitions is a strategic concern among firms as it relates to their success or downfall. Yet the analysis can become difficult as the network of firms increase, with each one having a different set of interactions and strategies. Our approach would be to consider the simplest system and investigate its critical transitions. With only two firms competing, duopoly is the simplest form of oligopoly. ${ }^{9}$ Even so, it allows for complex interactions depending on the context of the competition and the set-up of the firms ${ }^{10,11}$ As such, different forms have been intensively studied to gain insight into the dynamics of industrial organizations. ${ }^{12}$

In this paper, we consider a heterogeneous quantity competition of firms each adjusting their production in a logistic process. We show that the resulting dynamics is governed by Lotka-Volterra equations. We extract the critical points and investigate the dominant eigenvalues around these points. We then identify conditions that critical slowing down can provide early warning prior to critical transitions in the dynamic duopoly.

\section{Competition Model}

Consider two firms 1 and 2 each with a product competing for the same market. The value of their respective product, as given by its price, depend on the quantities $\left(q_{1}, q_{2}\right)$ in the market, i.e. $p_{i}\left(q_{1}, q_{2}\right), i=1,2$. Expanding up to first order one obtains:

$$
p_{i}\left(q_{1}, q_{2}\right)=p_{i}(0,0)+q_{1} \frac{\partial p_{i}}{\partial q_{1}}+q_{2} \frac{\partial p_{i}}{\partial q_{2}} .
$$

Substituting $\alpha_{i}=p_{i}(0,0)$ and assuming the price decreases with quantity such that $\partial p_{i} / \partial q_{i}=-\beta_{i}$ and $\partial p_{i} / \partial q_{j}=-\gamma_{i j}, i \neq j=1,2$ we have:

$$
p_{i}\left(q_{1}, q_{2}\right)=\alpha_{i}-\beta_{i} q_{i}-\gamma_{i j} q_{j} .
$$

One can associate $\alpha$ as the quality of the product in the sense that the higher it is, the greater the product is valued. Rewriting $\beta_{i}=\left(\partial q_{i} / \partial p_{i}\right)^{-1}$, the parameter is inversely related to the elasticity concept in economics where one is concerned with the change in the quantities given the change in the price. ${ }^{13}$ On the other hand $\gamma_{i j}=\left(\partial q_{j} / \partial p_{i}\right)^{-1}$ captures how different the products are from each other. ${ }^{14}$ For instance, if $\gamma_{i j}>0$, the product $j$ adversely affects the value of product $i$ and as such can be considered a substitute to product $i$. For $\gamma_{i j}<0$, product $j$ increases 
the value of product $i$ and hence a complement to it; $\gamma_{i j}=0$ means that product $j$ has no impact on the value of product $i$.

With the market condition above, we now look at the profit of firm $i$. The profit $\pi_{i}\left(q_{i}\right)$ in producing and selling $q_{i}$ units would be the difference between its revenue and production cost. Its revenue in selling $q_{i}$ units of the product at price $p_{i}$ is $p_{i} q_{i}$. For zero fixed cost and constant marginal cost $m_{i}$, the total cost in production is $m_{i} q_{i}$. As such the profit would be given by:

$$
\pi_{i}\left(q_{i}\right)=\left(\alpha_{i}-m_{i}-\beta_{i}-\gamma_{i j} q_{j}\right) q_{i}
$$

For the firm to maximize its profit, it needs to find the quantity $q_{i}^{B}$ that would maximize (3). Taking $\partial \pi_{i} / \partial q_{i}$ and equating it to zero and following Cournot's conjecture that the other firm keeps its quantity constant $\left(\partial q_{j} / \partial q_{i}=0\right)$ (Cournot, 1960), we obtain the best response of firm $i$ given the output of firm $j$ :

$$
q_{i}^{B}\left(q_{j}\right)=\frac{\alpha_{i}-m_{i}-\gamma_{i j} q_{j}}{2 \beta_{i}} .
$$

Consider now the situation where the current output $q_{i}(t)$ is different from the best response $q_{i}^{B}\left(q_{j}\right)$ as given in (4). The firm then needs to have an adjustment process that will bring its production to the desired level. Here we consider an adjustment process that is given by a logistic equation:

$$
\frac{d q_{i}(t)}{d t}=k_{i}\left(q_{i}^{B}\left(q_{j}\right)-q_{i}(t)\right) q_{i}(t)
$$

where $k_{i}$ controls the speed of the adjustment. ${ }^{16}$ The larger $k$ is, the steeper the firm has to ramp or reduce its current output to meet the desired level.

Given the adjustment process above, the resulting dynamical equations become:

$$
\frac{d q_{i}(t)}{d t}=k_{i} q_{i}(t)\left(M_{i}-q_{i}(t)-c_{i j} q_{j}(t)\right)
$$

where

$$
\begin{aligned}
& M_{i}=\frac{\alpha_{i}-m_{i}}{2 \beta_{i}} \\
& c_{i j}=\frac{\gamma_{i j}}{2 \beta_{i}} .
\end{aligned}
$$

Equation (6) is formally equivalent to the Lotka-Volterra competitive equations. The maximum output of firm $i$ will be realized when firm $j$ ceases to produce (i.e. $\left.q_{j}=0\right)$. This monopoly output $M_{i}$ is just the carrying capacity of the firm and is proportional to the firm's net advantage $\left(\alpha_{i}-m_{i}\right)$. One can extract as well the competion coefficient $c_{i j}$ and is proportional to the differentiation of the products $\left(\gamma_{i j}\right)$. 


\section{Critical Transitions}

Given the dynamics above, we now investigate the equilibrium states and transitions near them. The model above has the following equilibrium points, eigenvalues and stability conditions.

Table 1. Equilibria, eigenvalues and stability conditions of the model.

\begin{tabular}{lll}
\hline Equilibrium Points $\left(q_{1}^{*}, q_{2}^{*}\right)$ & Eigenvalues & Stable if \\
\hline$(0,0)$ & $\lambda_{1}=k_{1} M_{1} ; \lambda_{2}=k_{2} M_{2}$ & Never \\
$\left(M_{1,0}\right)$ & $c_{21}>\frac{M_{2}}{M_{1}}$ \\
& $\lambda_{1}=-k_{1} M_{1}$ & \\
& $\lambda_{2}=-k_{2}\left(c_{21} M_{1}-M_{2}\right)$ & $c_{12}>\frac{M_{1}}{M_{2}}$ \\
& $\lambda_{1}=-k_{2} M_{2}$ & \\
& $\lambda_{2}=-k_{1}\left(c_{12} M_{2}-M_{1}\right)$ & $c_{12} c_{21}<1$ \\
$\left(\frac{M_{1}-c_{12} M_{2}}{1-c_{12} c_{21}}, \frac{M_{2}-c_{21} M_{1}}{1-c_{12} c_{21}}\right)$ & $\lambda_{ \pm}=\frac{-(a+b) \pm \sqrt{(a+b)^{2}-4\left(1-c_{12} c_{21}\right) a b}}{2}$ & \\
& $a=k_{1} q_{1}^{*} ; b=k_{2} q_{2}^{*}$ & \\
\hline
\end{tabular}

The first equilibrium point is the trivial case where both firms do not produce and this condition is never stable as the eigenvalues are both positive. The next two results in the persistence of one firm and the demise of the other. This condition is stable if the impact of one firm on the other, as given by the competition coefficient, is larger than the ratio between the firms' monopoly output. The last equilibrium point pertain to the coexistence of the firms. This is stable if the impact of each firm is less than the ratio of their monopoly outputs.

Now we investigate the transition from one dynamical state to another and see how the equilibrium values change as one parameter is varied. Suppose changes in the business condition alters the advantage of firm 1 but have no effect on firm 2 . This can be a change in its quality or cost structure (or both) resulting in a change in $M_{1}$. For $M_{1}$ very small compared to $M_{2}$, firm 2 would eventually monopolize the market. However as $M_{1}$ improves it would be possible to coexist with firm 2 . The value of $M_{1}$ that would allow this and the type of coexistence depends on the kind of competition.

For $c_{12} c_{21}>1$, a path dependent hysteresis-like phenomenon occurs. For smaller values of $M_{1}$, firm 2 would be the only one left in the long-term evolution of the competition. However, past $c_{12} M_{2}$ it will trade places with firm 2 - firm 1 now monopolizing the market. Reversing the direction, for high values of $M_{1}$, firm 1 will be the monopoly until it decreases below $M_{2} / c_{21}$ where firm 2 becomes the monopoly. In between $M_{2} / c_{21}$ and $c_{12} M_{2}$, an unstable coexistence (dotted lines) prevails with the outcome depending on their initial conditions. These features are shown in Figure 1.a.

For $c_{12} c_{21}<1$, a stable coexistence occurs when $M_{1}>c_{12} M_{2}$. Improving beyond $M_{2} / c_{21}$ results in firm 1 monopolizing the market. The graph of the equilibrium values as a function of $M_{1}$ is depicted in Figure 1.b. 


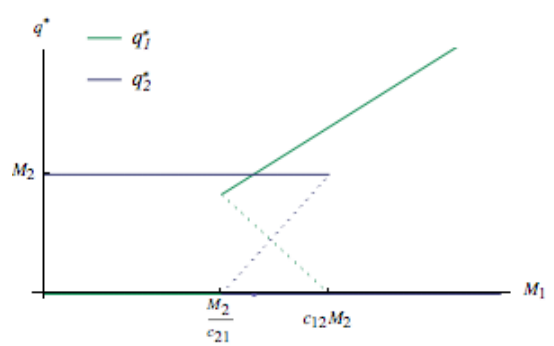

a.) $c_{12} c_{21}>1$

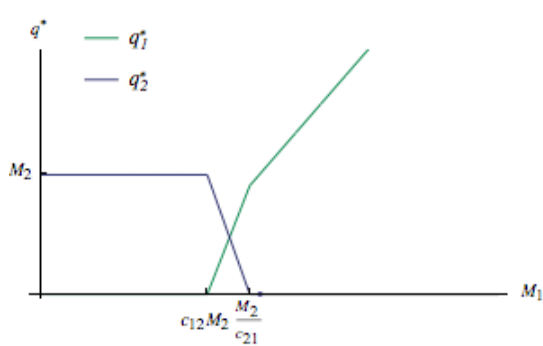

b.) $c_{12} c_{21}<1$

Fig. 1. (Color online) Equilibrium values as a function of $M_{1}$ for firm 1 and firm 2 .

\section{Critical Slowing Down}

Given the transitions above, of interest would be to find a way the transitions can be anticipated. To go about this, we take a look at the characteristic return time required for the system from an initial state $q_{i}\left(t_{o}\right)$ to a later state $q_{i}\left(t_{f}\right)$ as a function of the monopoly output $M_{1}$. From (6), this would be given by:

$$
T_{i}\left(M_{i}\right)=\int_{t_{o}}^{t_{f}} d t=\frac{1}{k_{i}} \int_{q_{i}}^{q_{f}} \frac{d q_{i}}{q_{i}\left(M_{i}-q_{i}-c_{i j} q_{j}\right)}
$$

For instance, for $M_{1}$ smaller than $c_{12} M_{2}$, the equilibrium state would have $q_{1}^{*}=0$ and $q_{2}^{*}=M_{2}$. However as $M_{1}$ increases we can estimate the return time from a very small value $\varepsilon$ to a quantity $q_{f}$

$$
\begin{aligned}
T_{1}\left(M_{1}\right) & =\frac{1}{k_{1}} \int_{\varepsilon}^{q_{f}} \frac{d q_{i}}{q_{1}\left(M_{1}-q_{1}-c_{12} M_{2}\right)} \\
& =\frac{1}{k_{1}\left(M_{1}-c_{12} M_{2}\right)} \ln \left(\frac{\varepsilon\left(q_{f}-M_{1}+c_{12} M_{2}\right)}{q_{f}\left(\varepsilon-M_{1}+c_{12} M_{2}\right)}\right) .
\end{aligned}
$$

On the other side of the critical point, the equilibrium state would be $\left(q_{1}^{*}, q_{2}^{*}\right)=$ $\left(\frac{M_{1}-c_{12} M_{2}}{1-c_{12} c_{21}}, \frac{M_{2}-c_{21} M_{1}}{1-c_{12} c_{21}}\right)$. As such coming from $q_{1}^{*}+\varepsilon$ to $q_{f}$ would take about:

$$
\begin{aligned}
T_{1}\left(M_{1}\right) & =\frac{1}{k_{1}} \int_{q_{1}^{*}+\varepsilon}^{q_{f}} \frac{d q_{i}}{q_{1}\left(M_{1}-q_{1}-c_{12} q_{2}^{*}\right)} \\
& =\frac{1}{k_{1}\left(M_{1}-c_{12} q_{2}^{*}\right)} \ln \left(\frac{\left(q_{1}^{*}+\varepsilon\right)\left(q_{f}-M_{1}+c_{12} q_{2}^{*}\right)}{q_{f}\left(q_{1}^{*}+\varepsilon-M_{1}+c_{12} q_{2}^{*}\right)}\right) .
\end{aligned}
$$

Plotting the return time as a function of $M_{1}$ yields the graph in Figure 2. 


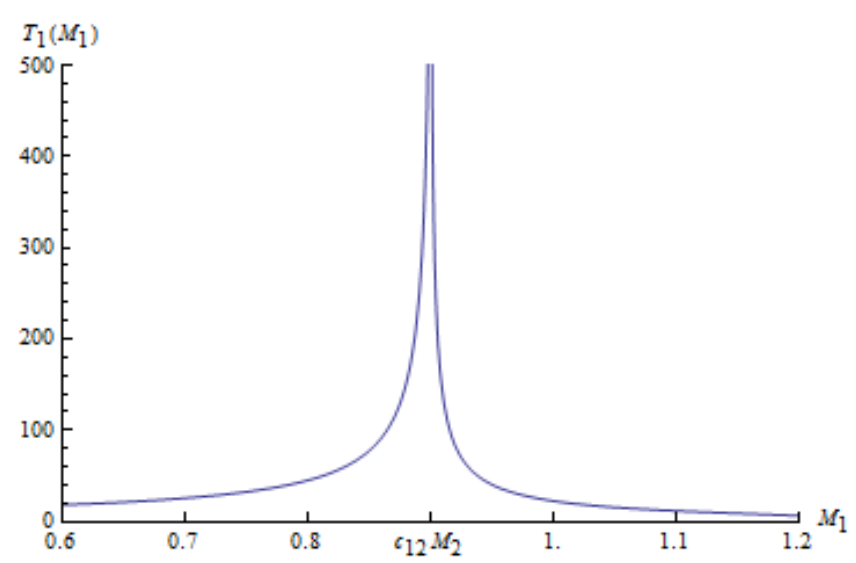

Fig. 2. Return time as a function of $M_{1} \cdot\left(M_{2}=12, c_{12}=c_{21}=075, q_{f}=075, \varepsilon=0001\right)$.

Evident in the graph is the rapid increase in the return time as the control parameter $M_{1}$ approaches the critical point $c_{12} M_{2}$. This critical slowing down on both directions comes before the actual critical transition.

A more general process of anticipating critical transitions using the concept of critical slowing has been developed. ${ }^{17}$. The characteristic return time is obtained from the recovery rate $\rho$ which is just the absolute value of the real part of the dominant eigenvalue. For instance, consider the transition from $\left(M_{1}, 0\right)$ to $\left(M_{2}\right)$ for the case $c_{12} c_{21}>1$. From the eigenvalues in Table 1 .

$$
\rho=\left|\operatorname{Re}\left(\lambda_{\text {dominant }}\right)\right|=\min \left(k_{1} M_{1}, k_{2}\left(c_{21} M_{1}-M_{2}\right)\right) .
$$

Using Eq. (11), if $k_{1} M_{1}<k_{2}\left(c_{21} M_{1}-M_{2}\right)$ then $\rho_{1}=k_{1} M_{1} \cdot{ }^{18}$ This recovery rate would be true for $M_{1}>k_{2} M_{2} /\left(k_{2} c_{21}-k_{1}\right)$. Otherwise, $\rho_{1}=k_{2}\left(c_{21} M_{1}-M_{2}\right)$. As such as the control parameter $M_{1}$ decreases, the recovery rate is decreasing as well, slowing down and becoming zero at the critical transition $M_{2} / c_{21}$. Similarly, for the state $\left(M_{2}\right)$ the recovery rate $\rho_{2}=k_{2} M_{2}$ for $M_{1}<M_{2}\left(k_{1} c_{12}-k_{2}\right) / k_{1}$ and $\rho_{2}=k_{1}\left(c_{12} M_{2}-M_{1}\right)$ otherwise. We then can superimpose the recovery rates to the plot of the equilibrium values as shown in Figure 3.

Evident in the figure is the discontinuous change in the recovery rate prior to the critical transitions in the equilibrium values. For instance, for firm 2 the recovery rate has changed at $\left(c_{12}-k_{2} / k_{1}\right) M_{2}$ whereas the abrupt change in its equilibrium value happened at $c_{12} M_{2}$. One can then measure the absolute change in the control parameter $M_{1}$ before the critical transition sets in, which in this case would be $\left(k_{2} / k_{1}\right) M_{2}=r_{2} M_{2}$ where $r_{j}$ is the relative speed of adjustment of firm $j$ to firm $i$. The higher $r_{2}$ is, the greater $k_{2}$ is compared to $k_{1}$, and the "sooner" the early warning could be.

Similarly for firm 1, the abrupt end of its decline can be forewarned by its recovery rate. Before it declines to $M_{2} / c_{21}$, the recovery rate has undergone a discontinuous change at $M_{2} /\left(c_{21}-k_{1} / k_{2}\right)$. As such a decline of $k_{1} M_{2} / k_{2} c_{21}\left(c_{21}-k_{1} / k_{2}\right)$ more 


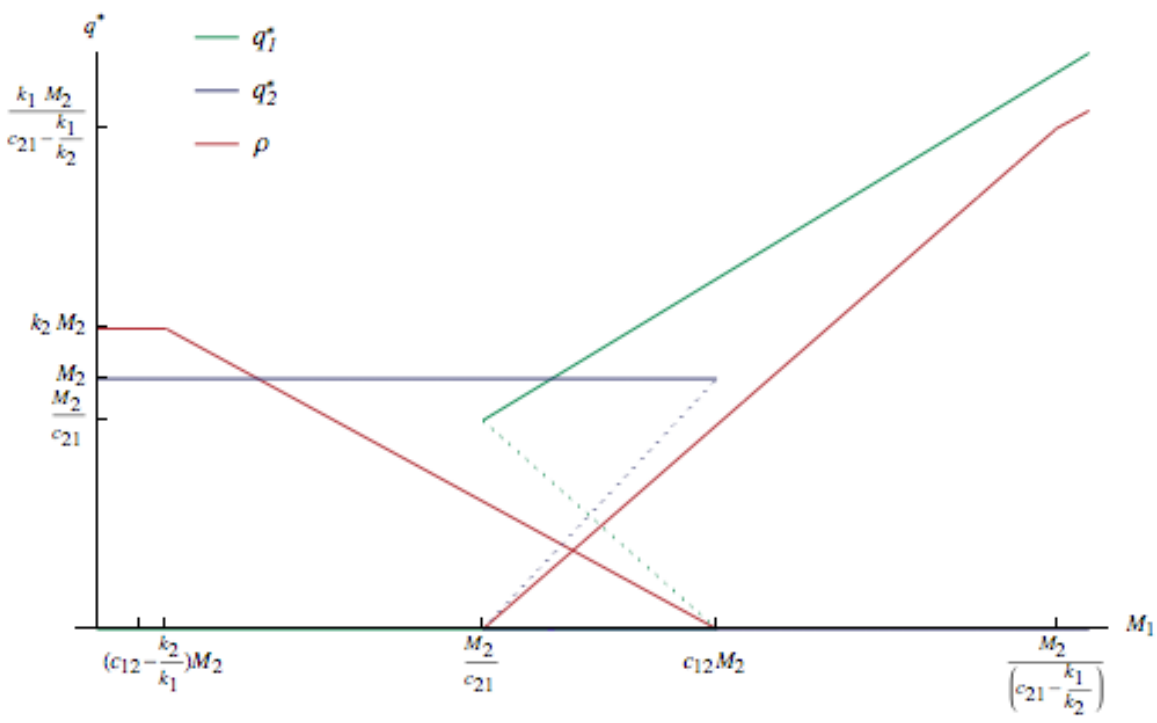

Fig. 3. (Color online) Equilibrium values $\left(q_{1}^{*}, q_{2}^{*}\right)$ and recovery rates $\rho$ for $c_{12} c_{21}>1$.

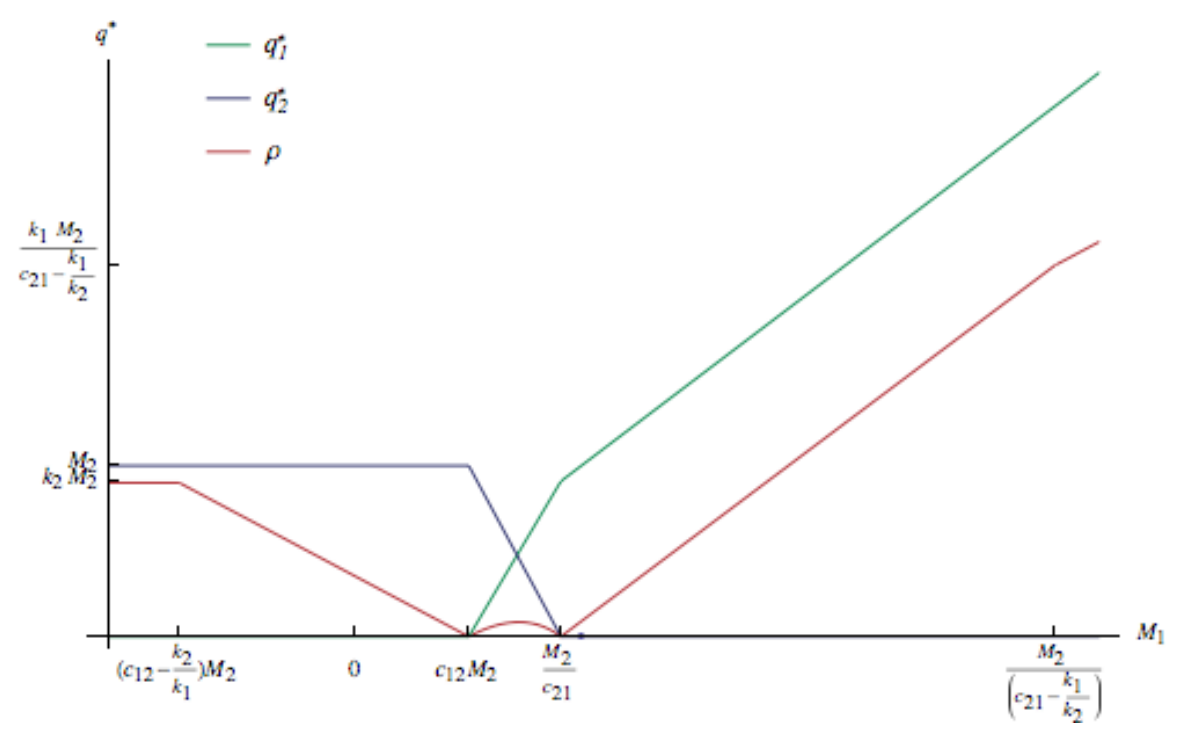

Fig. 4. (Color online) Equilibrium values $\left(q_{1}^{*}, q_{2}^{*}\right)$ and recovery rates $\rho$ for $c_{12} c_{21}<1$.

will bring it to the threshold of the critical transition. Rewriting to $r_{1} M_{2} / c_{21}\left(c_{21}-\right.$ $r_{1}$ ), the critical transition can be forewarned earlier if the competitive impact of firm 1 on 2 is low (i.e. low $c_{21}$ ) or the relative speed of adjustment $r_{1}$ is large $\left(k_{1}>k_{2}\right)$. 
For the case of $c_{12} c_{21}<1$, the presence or absence of a region for an early warning signal becomes more defined. Similar to the case of $c_{12} c_{21}>1$, the discontinuous transitions of the recovery rate occur at $\left(c_{12}-r_{2}\right) M_{2}$ for firm 2 and at $r_{1} M_{2} /\left(c_{21}-r_{1}\right)$ for firm 1 as shown in Figure 4. However, the presence of one precludes the other as an early warning signal as $r_{2}<c_{12}$ automatically means that $c_{21}-r_{1}<0$. And $c_{21}>r_{1}$ implies $r_{2}>c_{12}$ as illustrated in the figure below (i.e $\left(c_{12}-r_{2}\right) M_{2}<0$ ). As such, only the firm whose relative speed of adjustment is $r_{i}<c_{j i} i \neq j$ can have an early warning from its recovery rate.

\section{Conclusion}

Of interest from the preceding results is the role that the relative speed of adjustment $r_{i}=k_{i} / k_{j}$ plays in the presence or absence of an early warning signal. For $r_{1}$ very large, $c_{21}-r_{1}$ becomes negative and the discontinuous change of the recovery rate occurs at a negative monopoly output of firm 1 which is not realistic. Similarly, for large $r_{2}, c_{12}-r_{2}$ can become negative and firm 2 loses an early warning signal. As such, only at regions where $r_{i}<c_{j i}$ or $k_{i}<c_{j i} k_{j}$ does an early warning signal for both firms makes sense. Otherwise, the monopoly output shifts to negative and hence no longer feasible.

As such, it is possible to use the concept of critical slowing down to provide early warning to the critical transitions in a dynamic duopoly. The extent of the early warning depends on the monopoly output, competition coefficient and relative speed of adjustment. If the monopoly output is large, because, for instance of large firm advantage, the farther the warning is from a critical transition. The smaller the impact of the competition is, that is the smaller the competition coefficient, the more time one is forewarned. And the larger the relative speed of adjustment is, the sooner is the early warning.

\section{References}

1. M. Scheffer, J. Bascompte, W.A. Brock, V. Brovkin, S.R. Carpenter and V. Dakos, Early-warning signals for critical transitions, Nature 461, 53(2009).

2. M. Scheffer, S.R. Carpenter, T.M. Lenton, J. Bascompte, W. Brock, V. Dakos, J. van de Koppel, I.A. van de Leemput, S.A. Levin, E.H. van Nes, M. Pascual and J. Vandermeer, Anticipating Critical Transitions, Science 338, 344(2012).

3. C. Wissel, A universal law of characteristic return time near thresholds, Oecologia $\mathbf{6 5}$, 101(1984).

4. J.R. Tredicce, G.L. Lippia, P. Mandel, B. Charasse, A. Chevalier and B. Picque, Critical slowing down at a bifurcation, American Journal of Physics 72, 799(2004).

5. J. Lim and B.I. Epureanu, Forecasting a class of bifurcations: Theory and experiment, Physical Review E 83, 016203(2011).

6. C. Diks and C. Hommes, Early warning signals for critical transitions in finance, (Royal Economic Society Annual Conference, London, 2013).

7. D. Sornette, Critical market crashes, Physics Reports 378, 1(2003).

8. R.E. Farmer, The stock market crash of 2008 caused the Great Recession: Theory and evidence, Journal of Economic Dynamics and Control 36, 693(2012). 
9. J.W. Friedman, Oligopoly Theory, (Cambridge University Press, Cambridge, MA 1983).

10. M. Kopel, Simple and complex adjustment dynamics in Cournot duopoly models, Chaos, Solitons and Fractals 7, 2031(1996).

11. D. Rand, Exotic phenomena in games and duopoly models, Journal of Mathematical Economics 5, 173(1978).

12. J. Tirole, The theory of industrial organization (MIT Press, Cambridge, MA, 1988).

13. W. Mas-Collel, M.D. Green and J.R. Green, Microeconomic Theory (Oxford University Press, Oxford, 1995).

14. N. Singh and X. Vives, Price and Quantity Competition in a Differentiated Duopoly, The RAND Journal of Economics 15, 546(1984).

15. A.A. Cournot, Researches into the Mathematical Principles of the Theory of Wealth (Kelley, New York, 1960).

16. M.O. Escobido, Product interactions and industry shakeout, International Journal of Modern Physics: Conference Series 17, 104(2012).

17. E.H. van Nes and M. Scheffer, Slow recovery from perturbations as a generic indicator of a nearby catastrophic shift, American Naturalist 169, 738(2007).

18. R.A. Chisholm and E. Filotas, Critical slowing down as an indicator of transitions in two-species models, Journal of Theoretical Biology 257, 142(2009). 